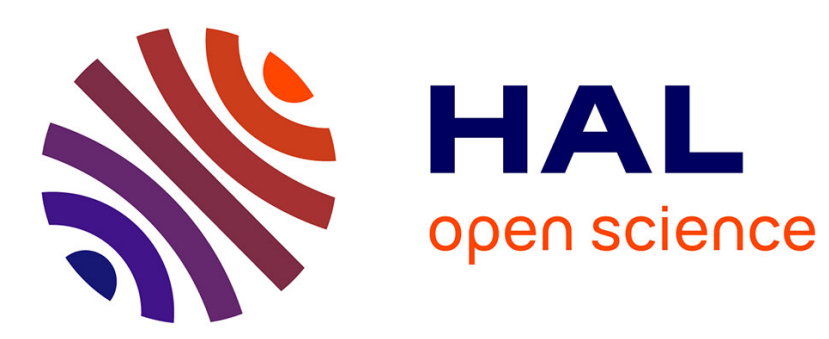

\title{
Non-linear numerical simulation of coiling by elastic finite strain model
}

\author{
Daniel Weisz-Patrault, Alain Ehrlacher, Nicolas Legrand, Elliette Mathey
}

\section{To cite this version:}

Daniel Weisz-Patrault, Alain Ehrlacher, Nicolas Legrand, Elliette Mathey. Non-linear numerical simulation of coiling by elastic finite strain model. Key Engineering Materials, 2015, 651-653, pp.1060-1065. 10.4028/www.scientific.net/KEM.651-653.1060 . hal-01275176

\section{HAL Id: hal-01275176 https://hal.science/hal-01275176}

Submitted on 2 Mar 2016

HAL is a multi-disciplinary open access archive for the deposit and dissemination of scientific research documents, whether they are published or not. The documents may come from teaching and research institutions in France or abroad, or from public or private research centers.
L'archive ouverte pluridisciplinaire HAL, est destinée au dépôt et à la diffusion de documents scientifiques de niveau recherche, publiés ou non, émanant des établissements d'enseignement et de recherche français ou étrangers, des laboratoires publics ou privés. 


\title{
Non-linear numerical simulation of coiling by elastic finite strain model
}

\author{
Daniel Weisz-Patrault ${ }^{1, \mathrm{a}}$, Alain Ehrlacher ${ }^{2, \mathrm{~b}}$, Nicolas Legrand ${ }^{3, \mathrm{c}}$ \\ and Eliette Mathey ${ }^{3, \mathrm{~d}}$ \\ ${ }^{1}$ Laboratoire de Mécanique des Solides, École Polytechnique, 91128 Palaiseau cedex, France \\ ${ }^{2}$ Laboratoire Navier, Ponts ParisTech, 6 \& 8 Ave Blaise Pascal, 77455 Marne La Vallee, France \\ ${ }^{3}$ ArcelorMittal Global R\&D, Voie Romaine BP 30320, 57283 Maizières-lès-Metz cedex, France \\ a'weisz@Ims.polytechnique.fr, balain.ehrlacher@enpc.fr, 'cnicolas.legrand@arcelormittal.com, \\ deliette.mathey@arcelormittal.com
}

Keywords: Non-linear simulation, Contact, Coiling process, Finite strain, Multiplicative elasticity

\begin{abstract}
The coiling process under traction is considered, with an incoming residual stress profile (that can be sufficiently compressive to make the strip buckle): a flatness defect. This paper details a 3D non-linear numerical simulation taking into account the contact of the strip on itself, with a perfect contact law. The model relies on elastic behavior at finite strain because of large rotations. Even though the behavior is elastic, the yield Von Mises criterion is computed and gives information about flatness defects (plastic zones are approximated by zones where the yield stress is exceeded). Furthermore, the paper aims at very short computation times. The modeling strategy relies (for each time step) on two analytical sub-steps. Numerical minimization procedure is used in order apply weak boundary conditions. Results are discussed with respect to a comprehensive Finite Element simulation and good agreement is observed.
\end{abstract}

\section{Introduction}

This paper focuses on a numerical strategy for modeling efficiently the winding of the strip on itself. The main purpose is to develop a fast tool that enables to develop coiling strategies that take into account residual stress evolution. Classical winding defects detailed in [1] are not broached in this contribution. Prior coiling models are overviewed in [2]. In addition a simple elastic modeling is developed in [3]. Most winding models rely on the same assumptions and very similar methods are detailed in [2] and [3]: layers are modeled as concentric rings loaded by internal pressure, thus stresses induced by the curvature of the strip are not taken into account. Then elastic thin walled-theory is used, therefore radial and tangential stress fields are homogeneous across the strip thickness. There is no explicit contact formulation, which is taken into account as a post-processing of a linear elastic calculation. In contrast, the winding model presented here does not rely on concentric rings. Radius of curvature and contact length of the strip on itself vary all along each cycle and are determined by mechanical computations. Thus, a spiral is described instead of concentric rings. Stresses induced by the strip curvature are taken into account and multiplicative elasticity formalism (finite strain) is used because of large rotations. The model is incremental and each time step is subdivided into two different sub-steps. The first one consists in bending a flat incoming infinitesimal strip portion with an unknown radius, the second sub-step consist in making contact with the rest of the coil underneath with an unknown pressure. Both sub-steps are analytical and an explicit relationship between the unknown radius of curvature and the unknown contact pressure is obtained with a perfect contact law (i.e., without roughness, interpenetration of surfaces is not allowed). Therefore, the contact pressure is determined as a function of the unknown radius of curvature, which is finally determined with weak boundary conditions, which ensure that the resultant traction matches the imposed time-dependent resultant traction. A minimization process performs the latter computation numerically. 


\section{Model description}

Reference configuration. The Cartesian basis is denoted by $\left(\boldsymbol{e}_{\boldsymbol{X}}, \boldsymbol{e}_{\boldsymbol{Y}}, \boldsymbol{e}_{\boldsymbol{Z}}\right)$ and the associated coordinates are $(X, Y, Z)$. The rolling direction is $\boldsymbol{e}_{\boldsymbol{X}}$, the direction along the strip thickness is $\boldsymbol{e}_{\boldsymbol{Y}}$ and the direction along the strip width or the mandrel axis is $e_{Z} . L$ denotes the half width of the strip. The observer is fixed to the mandrel. The latter does not rotate and the strip winds around the mandrel. Polar coordinates $(r, \theta, z)$ are used for the description of the strip in the actual configuration, as shown in Fig. 1. The part of the strip already wound is clearly defined by $\theta \in[0,-\omega t]$ (where $\omega$ is the rotation speed) and the remaining part is submitted to a rigid rotation. It should be noted that $\theta$ is negative and strictly decreasing during the coiling process.
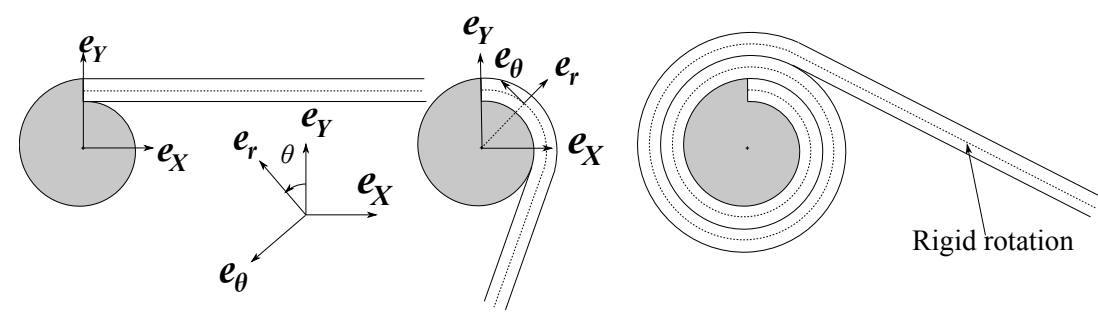

Fig. 1: Actual configuration: observer fixed to the mandrel

Residual stress. Previous processes such as rolling process and run out table are responsible for significant residual deformations which are not compatible, thus an elastic field is needed so that the total deformation is compatible leading to residual stresses. In this paper it is assumed that the residual stress field is prevailing according to $\boldsymbol{e}_{\boldsymbol{X}} \otimes \boldsymbol{e}_{\boldsymbol{X}}$ and the other components are neglected. Thus the residual stress tensor is introduced $\underline{\Pi}^{(0)}=\Pi_{X X}^{(0)}(X, Y, Z) \boldsymbol{e}_{\boldsymbol{X}} \otimes \boldsymbol{e}_{\boldsymbol{X}}$. For each section the equilibrium is guaranteed. An elastic tensor $\underline{\boldsymbol{E}}^{(0)}$ is associated (via the isotropic elastic behavior) to the residual stress profile and $\underline{\boldsymbol{E}}^{(0)}=E_{0} \boldsymbol{e}_{\boldsymbol{X}} \otimes \boldsymbol{e}_{\boldsymbol{X}}+\widetilde{E}_{0}\left(\boldsymbol{e}_{\boldsymbol{Y}} \otimes \boldsymbol{e}_{\boldsymbol{Y}}+\boldsymbol{e}_{\boldsymbol{Z}} \otimes \boldsymbol{e}_{\boldsymbol{Z}}\right)$ with $J_{0}=1+\Pi_{X X}^{(0)} /\left(3 k_{0}\right)$, $\widetilde{E}_{0}=\sqrt{J_{0} / E_{0}}$ and where $E_{0}$ is the only real root of $E_{0}^{3}-E_{0} J_{0}^{\frac{5}{3}} \Pi_{X X}^{(0)} / \mu_{0}-J_{0}=0$. Shear and bulk moduli are denoted respectively by $\mu_{0}$ and $k_{0}$.

Time as a space variable. Let $X_{\max }: t \mapsto X_{\max }(t)$ denote the maximal strip length in the reference configuration, which is wound at instant $t$. The notation $X_{\max }$ is related to the value of $X$ such as $\theta=$ $-\omega t$. Time variable $t$ can be substituted by a space variable $X_{\max }(t)$. In the following, $X_{\max }$ is used instead of $X_{\max }(t)$. So the wound part of the strip is described by $X \in\left[0, X_{\max }\right]$. The infinitesimal strip portion being wound is described by $X=X_{\max }$ and $X+\mathrm{d} X$.

Assumptions. The model relies on some assumptions, which are specified here. $A 1$ : Once in contact, slips between layers are not allowed. $A 2$ : Cross sections in the reference configuration are transformed into cross sections of the actual configuration. (" $X=$ Constant" transformed into " $\theta=$ Constant"). Therefore, the angle representing a particle in the actual configuration depends only on $X$. $A 1$ enables to discard the time dependence and $A 2$ enables to discard the $Y$ and $Z$ dependencies. A3: Plain strain assumption is made. Planes " $Z=$ Constant" in the reference configuration are transformed in the actual configuration in the same planes. $A 4$ : For each time step, the infinitesimal strip portion added to the rest of the coil is assumed to have the same traction profiles at sections $X$ and $X+\mathrm{d} X$. Thus, this model describes piecewise constant traction profiles according to the $X$ variable. Therefore, for each infinitesimal strip portion added to the coil, displacements are purely radial.

Modeling steps. As mentioned above, the present model is incremental and for each time step an infinitesimal strip portion is curved and put in contact with the rest of the coil. The strategy consists in dividing each time step in two different sub-steps. Since the problem is highly non linear (contact problem and finite strain formalism), the deformation path matters and other modeling choices could lead to different results. The infinitesimal strip portion at section $X$ in the reference configuration 
lying between sections $X=X_{\max }$ and $X+\mathrm{d} X$ is considered. The model describes how the strip portion is curved and then how the contact with the rest of the coil is performed.

Step 1. The infinitesimal strip portion is curved arbitrarily with a trial radius of curvature $R^{*}(X)(Z$ independent). Thus, this step correspond to a global curvature of the strip portion regardless to the axial position $Z$, which will be corrected in step 2 . The trial radius $R^{*}(X)$ will be determined by applying weak boundary conditions in the end. This step involves large rotations and multiplicative elastic formalism is used. The mid-plane of the infinitesimal strip portion is transformed into a cylindrical cylinder of radius $R^{*}(X)$. The transformation $\boldsymbol{\Phi}^{(1)}(X, Y, Z)=\left(R^{*}(X)+Y\right) \boldsymbol{e}_{\boldsymbol{r}}+Z \boldsymbol{e}_{\boldsymbol{Z}}$ is imposed. After the transformation $\Phi^{(1)}$, the upper-plane has the radius $R^{*}(X)+\delta(Z)$ and the lower plane has the radius $R^{*}(X)-\delta(Z)$. This is obtained mostly by applying bending moments at both sections $X$ and $X+\mathrm{d} X$. These bending moments are due to traction profiles through the strip thickness (i.e., $e_{Y}$ direction). In the following, the associated Cauchy stress tensor $\underline{\sigma}^{(1)}$ is evaluated as a function of radius $R^{*}(X)$. Since the transformation $\Phi^{(1)}$ is imposed, unwanted body forces $f_{b}$ are introduced and calculated with $\operatorname{div} \underline{\boldsymbol{\sigma}}^{(1)}=-f_{b}$. However, the radial stress $\sigma_{r r}^{(1)}$ do not vanish at the upper and lower surfaces of the strip portion, and the resultant force of unwanted body forces compensates the unwanted resultant force of residual surface traction. Therefore, a global equilibrium is ensured through the strip portion thickness.

Step 2. The contact between the curved strip portion at section $X$ and the rest of the coil is modeled, assuming a perfect no sliding contact. The strip portion is pressed against the coil upper surface at section $X_{-}$(defined implicitly by $\theta\left(X_{-}\right)=\theta(X)+2 \pi$ ). The radius $R^{*}(X)-\delta(Z)$ of the lower surface of the strip portion at section $X$ at the end of step 1 and the radius $r_{c}\left(X_{-}, Z\right)$ of the coil upper surface at section $X_{-}$are geometrically incompatible. Therefore the contact leading to the compatibility of both radii imposes a pressure denoted by $P^{*}(X, Z)$ at the interface, which is small enough, so that step 2 is an additive correction of step 1 under infinitesimal strains assumption. This pressure field is parametrized by the trial radius of curvature $R^{*}(X)$. Displacements associated to the contact pressure $P^{*}(X, Z)$ that make radii compatibles should be calculated in order to establish a relationship between $P^{*}(X, Z)$ and $R^{*}(X)$. For each axial position $Z$, displacements of the infinitesimal strip portion for a fixed section $X$ can be computed easily analytically using $A 4$ implying purely radial displacements. The radial displacement of the strip portion for this step is denoted by $U^{(2 a)}(X, Y, Z)$. Likewise, displacements of the rest of the coil at section $X_{-}$are purely radial and can be computed analytically and are denoted by $U^{(2 b)}(X, Z)$. Since displacements are purely radial the radius of curvature is changed only for all $X^{\prime}$ aligned underneath $X$ along the radial direction (i.e., $\theta\left(X^{\prime}\right)=\theta(X)+2 k \pi$ with $k \in \mathbb{N}$ ). Hence the radius of the coil underneath the strip portion at the end of step 2: $r_{c}\left(X_{-}, Z\right)+U^{(2 b)}(X, Z)$.

Contact law. In this paper a perfect contact is modeled. As explained above, the radius of the infinitesimal strip portion lower-surface $R^{*}(X)-\delta(Z)$ and the radius of the rest of the coil uppersurface $r_{c}\left(X_{-}, Z\right)$ are not compatible. The unknown pressure $P^{*}(X, Z)$ enables to make them compatible. Therefore the radii up-dated by the radial displacements due to $P^{*}(X, Z)$ should coincide if $P^{*}(X, Z) \geq 0$. Thus the contact law can be written as follows:

$$
\left\{\begin{array}{l}
R^{*}(X)-\delta(Z)+U^{(2 a)}(X,-\delta(Z), Z)=r_{c}\left(X_{-}, Z\right)+U^{(2 b)}(X, Z) \text { and } P^{*}(X, Z) \geq 0 \\
R^{*}(X)-\delta(Z) \geq r_{c}\left(X_{-}, Z\right) \text { and } P^{*}(X, Z)=0
\end{array}\right.
$$

The first equation of Eq. 1 is the compatibility (equality) of radii for positive pressures. The second equation expresses the possibility of having no contact. Considering that both steps are solved analytically, the contact law enables to write the pressure field $P^{*}(X, Z)$ as an explicit analytical function of $R^{*}(X)$ (where $R_{1}=R^{*}(X)-\delta(Z)$ and $R_{2}=R^{*}(X)+\delta(Z)$ ):

$$
P^{*}(X, Z)= \begin{cases}\frac{r_{c}\left(X_{-}, Z\right)-R_{1}}{r_{c}\left(X_{-}, Z\right)} \frac{1}{2\left(\lambda_{0}+\mu_{0}\right)}+\frac{R_{1}^{2}}{2 \mu_{0}} \frac{\mu_{0}}{R_{2}^{2}-R_{1}^{2}}\left(\frac{R_{2}^{2}}{\lambda_{0}+\mu_{0}} R_{1}+\frac{R_{2}}{R_{1}}\right) & \text { if } \quad R_{1} \leq r_{c}\left(X_{-}, Z\right) \\ 0 & \text { if } \quad R_{1} \geq r_{c}\left(X_{-}, Z\right)\end{cases}
$$


Calculations. Simple calculations give the total stress field in the infinitesimal strip portion, denoted by $\underline{\boldsymbol{\sigma}}^{(1+2)}=\underline{\boldsymbol{\sigma}}^{(1)}+\underline{\boldsymbol{\sigma}}^{(2 a)}+\underline{\boldsymbol{\sigma}}^{(2 b)}$ (the additive formulation comes from the infinitesimal strains assumption made for step 2) where $J=\left(R^{*}(X)+Y\right) / R^{*}(X)$ and:

$$
\begin{aligned}
& \sigma_{r r}^{(1)}(X, Y, Z)=\frac{\mu_{0}}{3}\left(J J_{0}\right)^{-\frac{5}{3}}\left(\frac{J_{0}}{E_{0}}-\left(J E_{0}\right)^{2}\right)+k_{0}\left(J J_{0}-1\right) \\
& \sigma_{\theta \theta}^{(1)}(X, Y, Z)=\frac{\mu_{0}}{3}\left(J J_{0}\right)^{-\frac{5}{3}}\left(-2 \frac{J_{0}}{E_{0}}+2\left(J E_{0}\right)^{2}\right)+k_{0}\left(J J_{0}-1\right) \\
& \sigma_{z z}^{(1)}(X, Y, Z)=\frac{\mu_{0}}{3}\left(J J_{0}\right)^{-\frac{5}{3}}\left(\frac{J_{0}}{E_{0}}-\left(J E_{0}\right)^{2}\right)+k_{0}\left(J J_{0}-1\right)
\end{aligned} \quad \begin{gathered}
\sigma_{r r}^{(2 a)}(X, Y, Z)=P^{*}(X, Z) \frac{R_{1}^{2}}{R_{2}^{2}-R_{1}^{2}}\left(1-\left(\frac{R_{2}}{R^{*}(X)+Y}\right)^{2}\right) \\
\sigma_{\theta \theta}^{(2 a)}(X, Y, Z)=P^{*}(X, Z) \frac{R_{1}^{2}}{R_{2}^{2}-R_{1}^{2}}\left(1+\left(\frac{R_{2}}{R^{*}(X)+Y}\right)^{2}\right)
\end{gathered}
$$

In addition $\underline{\boldsymbol{\sigma}}^{(2 b)}$ is the up-date of Cauchy stress in all previous layers under pressure $P^{*}(X, Z)$ then for all $X^{\prime}$ such as $\theta\left(X^{\prime}\right)=\theta(X)+2 k \pi, \sigma_{r r}^{(2 b)}\left(X^{\prime}, Z\right)=\sigma_{\theta \theta}^{(2 b)}\left(X^{\prime}, Z\right)=-P^{*}(X, Z)$. It should be noted that a rather thin discretization is needed along the axial direction $e_{Z}$ (500 points in this paper), in order to have accurate contact zone. There is no mesh along the $Y$ direction because if $\Pi_{X X}^{(0)}$ is given analytically all quantities are analytical with respect to $Y$.

Solution determination and boundary condition. By applying the contact law, the total stress field is determined as a function of $R^{*}(X)$. The trial radius of curvature $R^{*}(X)$ should be determined by adding some boundary conditions which are expressed in terms of resultant traction applied to the section $X$ of the infinitesimal strip portion and given by $T^{*}=\int_{-L}^{L} \int_{-\delta(Z)}^{\delta(Z)} \sigma_{\theta \theta}^{(1+2)}(X, Y, Z) \mathrm{d} Y \mathrm{~d} Z$. Indeed the coiling process is motorized and a known nominal traction denoted by $T^{a}(X)$ is applied to the strip. The radius of curvature $R(X)$ solution of the problem is determined when equality between $T^{a}$ and $T^{*}$ is satisfied, which can be written as a minimization problem: $R(X)=\underset{R^{*}(X) \geq 0}{\operatorname{argmin}}\left|T^{*}-T^{a}\right|$.

Von Mises criterion. The yield Von Mises criterion is computed in order to give insights with respect to plastic behavior of the strip, so that flatness defects evolution can be understood. Thus the yield Von Mises criterion (without kinematic hardening) is given by: $\sqrt{\frac{3}{2} \underline{s}^{(1+2)}: \underline{\boldsymbol{s}}^{(1+2)}}$, where the deviatoric stress tensor is $\underline{\boldsymbol{s}}^{(1+2)}=\underline{\boldsymbol{\sigma}}^{(1+2)}-\left(\operatorname{tr}\left(\underline{\boldsymbol{\sigma}}^{(1+2)}\right) / 3\right) \underline{\mathbf{1}}$. It should be compared with the yield stress $\sigma_{0}$. From purely elastic computations, the corresponding yield Von Mises criterion can be evaluated, and plastic zones can be roughly approximated.

\section{Validation}

This section is an attempt to validate the presented method by FEM (using Abaqus). Simulation parameters are listed in Table $1 \mathrm{~b}$ and mechanical properties are listed in Table 1a. The geometrical strip profile is parabolic with 20 elements through the strip width. Perfect contact law is used. The applied stress imposed on the strip is $\sigma_{T}=30 \mathrm{MPa}$. Furthermore, elastoplastic behavior without hardening (isotropic or kinematic) is considered with yield stress $\sigma_{0}=500 \mathrm{MPa}$. The flat strip is wound around a rigid finite cylinder. Computation times being extremely long (around 2 weeks) for this highly nonlinear problem, only 4 cycles are modeled with only 50 elements along the rolling direction for each cycle and 3 elements trough the thickness. Thus, stresses due to the curvature of the strip are not accurate. The order of magnitude is far below common estimations of the theory of shells. However, contact pressures and especially the effect of the geometrical profile of the strip are more accurate because contact depends mainly on surface geometries which are correctly described. Therefore the comparison between the Finite Element model and the presented model is done after shifting the origin of stress. Comparison of strip tension at $Y=1$ for two angular positions $\theta=\pi / 2$ and $\theta=3 \pi / 2$ are presented for the four cycles in Fig. 2 and good agreement is observed.

\section{First tests and results}

This fast non-linear simulation can be tested with various input parameters such as strip geometrical profile, applied traction, residual stress profile or mandrel's radius. Coiling parameters are listed in 
Table 1: Modeling parameters

(a) Mechanical properties

\begin{tabular}{|l|cc|c|}
\hline Young modulus & $E$ & $(\mathrm{MPa})$ & 210000 \\
\hline Poisson ratio & $\nu$ & $(-)$ & 0.3 \\
\hline Shear modulus & $\mu_{0}$ & $(\mathrm{MPa})$ & 80769 \\
\hline Bulk modulus & $k_{0}$ & $(\mathrm{MPa})$ & 175000 \\
\hline
\end{tabular}

(b) Simulation parameters
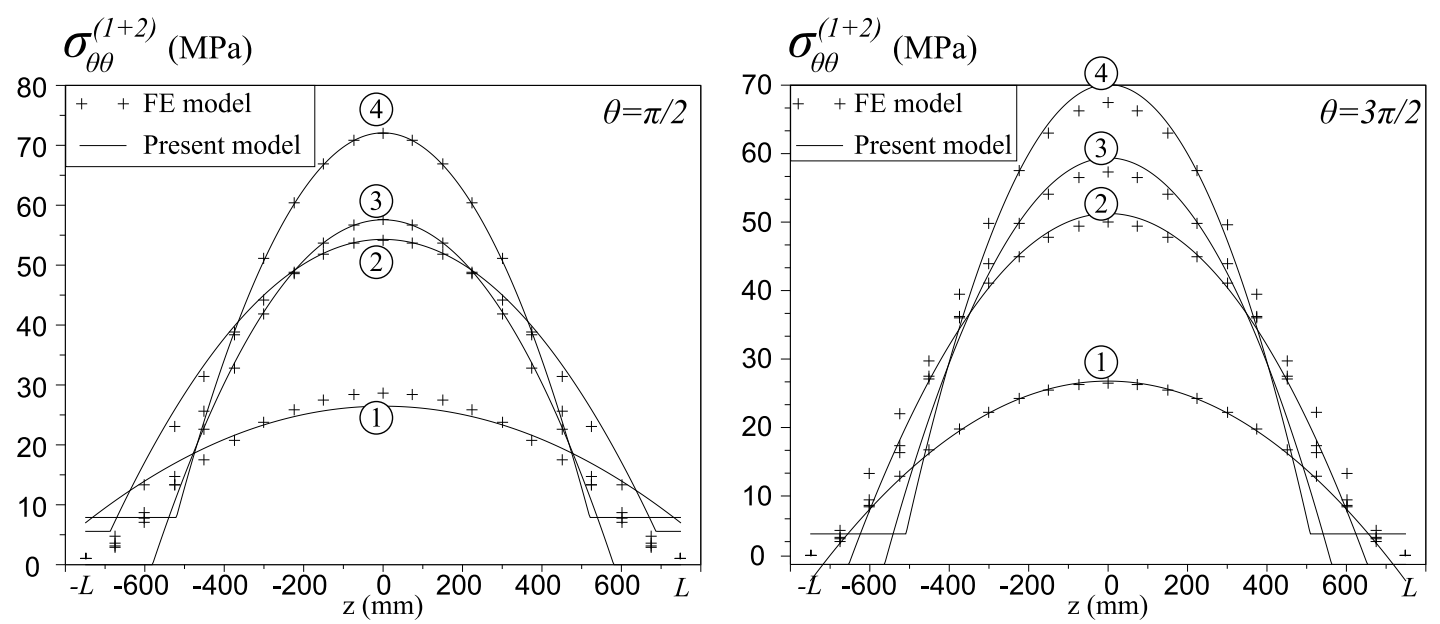

Fig. 2: FEM validation at $Y=1$ with Abaqus

Tables $1 \mathrm{~b}$ and $1 \mathrm{a}$. The number of modeled cycles is fixed to 100 and computed in around 5 seconds (time displayed by Scilab with a quadcore $2.8 \mathrm{GHz}$ ). Deformed geometry, contact length of the strip on itself, stresses and strains are computed in the whole coil for each time step.

Influence of residual stress profile. A long edges defect is modeled with a parabolic residual stress profile according to the axial direction $e_{Z}: \Pi_{X X}^{(0)}(X, Y, Z)=a+b\left(\frac{Z}{L}\right)^{2}$, where parameters $a$ and $b$ are zeros for Test 1 (no flatness defect) and $a=50$ and $b=-150$ for Test 2 (long edges defect). Total strip tension $\sigma_{\theta \theta}^{(1+2)}$ is presented for a few cycles at $Y=0$ for the last infinitesimal strip portion (i.e., $\theta(X)=-2 k \pi$ with $k \geq 1$ ) in Fig. 3. During winding, the contact length of the strip on itself decreases and $\sigma_{\theta \theta}^{(1+2)}$ increases so that the resultant force reaches the applied traction $T^{a}$. Furthermore, the residual stress profile is clearly identified where the contact is lost. At $Y=0$, stresses due to the curvature of the strip are negligible, however at $Y=1$ one can see the effect of bending, which decreases when the radius of curvature increases.

Influence of the applied traction. Contact lengths for the case without residual stress profile are represented in Fig. 4 for three applied resultant forces corresponding to 10, 30 and $90 \mathrm{MPa}$. Details are also given for the first cycles. It is clear that traction ensure a certain level of contact in the coil.

\section{Conclusions}

This paper presents a fast non-linear simulation of coiling process based on elasticity at finite strain. The contact of the strip on itself is perfect with no slips. During each time step an infinitesimal strip portion is wound on the coil. The model has been compared with a Finite Element simulation and good agreement is obtained. Very short computation times ( $5 \mathrm{sec}$ for 100 cycles: time displayed by Scilab with a quadcore $2.8 \mathrm{GHz}$ ) enables extensive parametric studies. This study gives interesting perspectives with respect to flatness control and coiling strategies. 

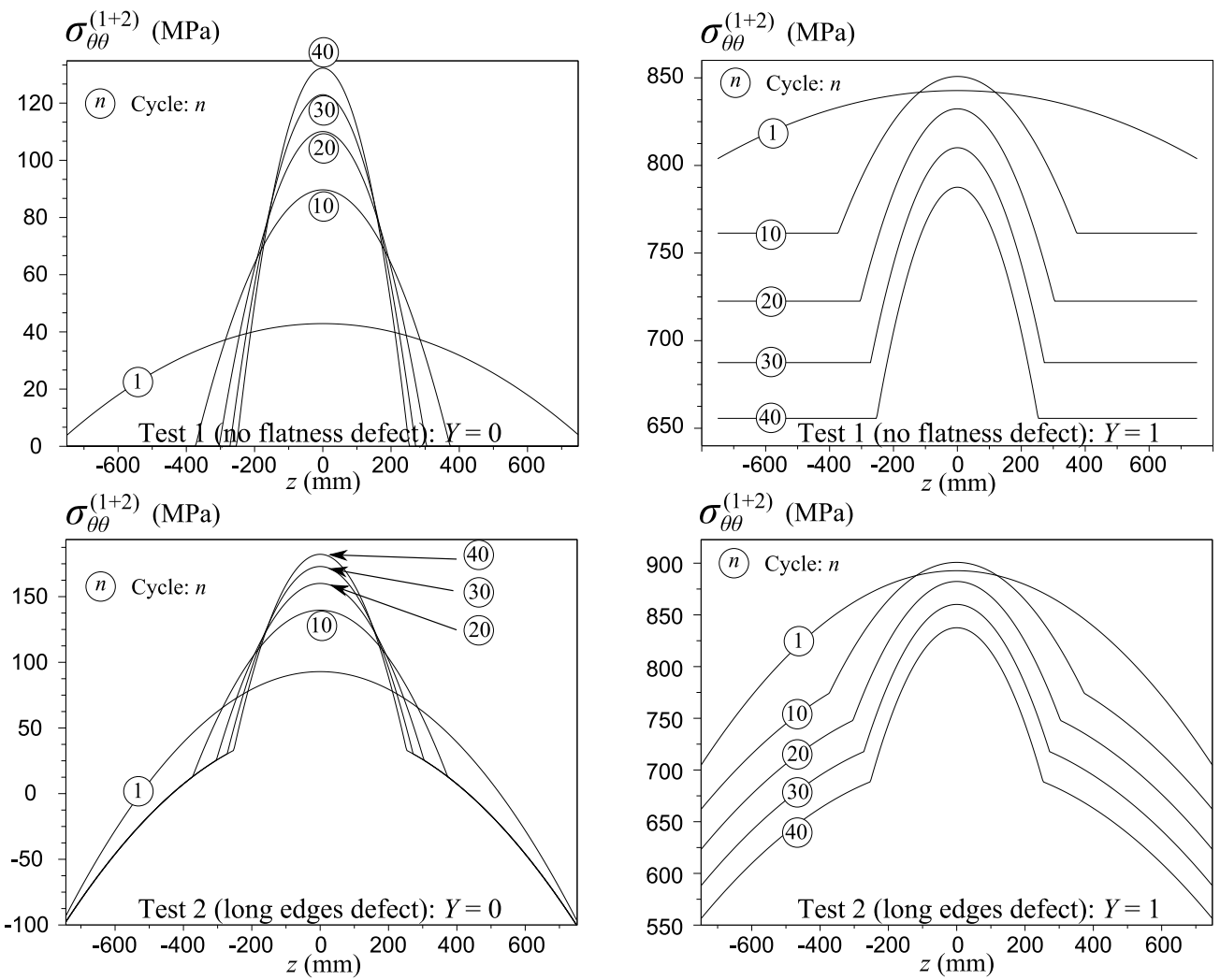

Fig. 3: $\sigma_{\theta \theta}^{(1+2)}$ profile along the axial direction
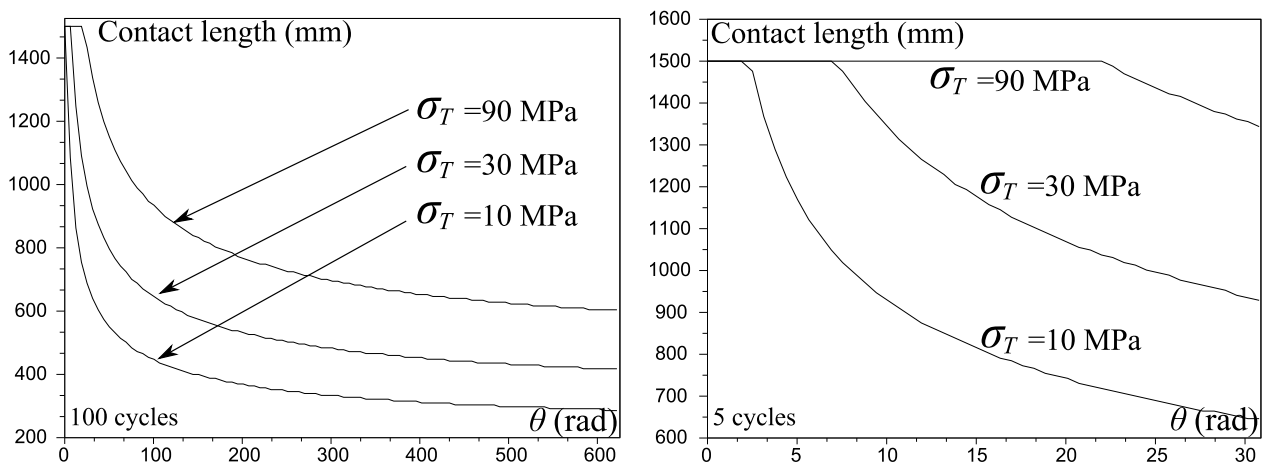

Fig. 4: Contact length as a function of the applied traction

\section{References}

[1] WJ Edwards and G Boulton. The mystery of coil winding. In 2001 Iron and Steel Exposition and AISE Annual Convention, page 2001, 2001.

[2] Jantzen L Hinton. A Study on the Effects of Coil Wedge During Rewinding of Thin Gauge Metals. $\mathrm{PhD}$ thesis, Wright State University, 2011.

[3] JM Hudzia, F Ferrauto, and P Gevers. Stress calculation applied to a coil, and optimization of coiling tension. Cah. Inf. Tech. Rev. Metall., 91(6):937-943, 1994. 